\title{
Really Simple Syndication
}

National Cancer Institute

\section{Source}

National Cancer Institute. Really Simple Syndication. NCI Thesaurus. Code C80149.

Various XML file formats suitable for disseminating real-time information via subscription on the Internet. 